\title{
ON THE SUMMABILITY OF CERTAIN SERIES FOR UNBOUNDED NONLINEAR FUNCTIONALS
}

\author{
R. H. CAMERON AND C. HATFIELD
}

1. Introduction. In a previous paper the authors investigated the infinite-dimensional Abel summability of the orthogonal development of a bounded nonlinear functional in terms of a set of closed orthonormal functionals. ${ }^{2}$ In this paper we want to remove the condition of boundedness on the functional. Our main theorem is as follows:

THEOREM. Let $C$ be the space of all continuous functions $x(t)$ on $0 \leqq t \leqq 1$ such that $x(0)=0$ and let $L_{2}(C)$ be the space of all Wienermeasurable functionals of summable square. Let $F(x)$ be a functional on $L_{2}(C)$ such that

$$
|F(x)| \leqq B \exp \left\{A \int_{0}^{1}[x(t)]^{2} d t\right\},
$$

and which is continuous in the Hilbert topology at $x_{0}$. Let

$$
\Psi_{m_{1}}, \cdots, m_{N}(x)=\prod_{k=1}^{N} H_{m_{k}}\left(\int_{0}^{1} 2^{1 / 2} \cos \left(\frac{2 k-1}{2}\right) \pi t d x(t)\right),
$$

where $H_{n}$ is the partially normalized Hermite polynomial

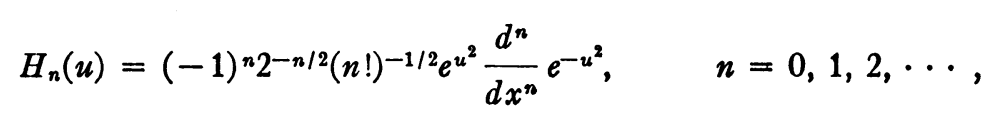

and let

$$
A_{m_{1}}, \cdots, m_{N}=\int_{C}^{w} F(x) \Psi_{m_{1}}, \cdots, m_{N}(x) d_{w} x .
$$

Then ${ }^{3}$

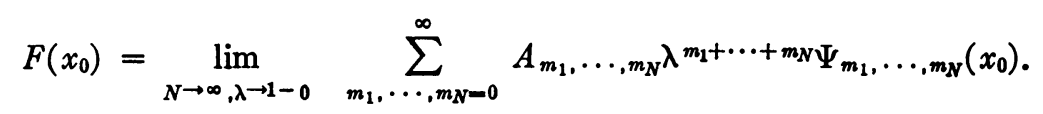

Presented to the Society, April 27, 1951; received by the editors August 29, 1952.

1 On the summability of certain orthogonal developments of nonlinear functionals, Bull. Amer. Math. Soc. vol. 55 (1949) pp. 130-145.

2 A simpler proof of this theorem has since been given by G. Maruyama, Kodai Mathematical Seminar Reports, no. 3, 1950, pp. 41-44.

${ }^{3}$ Here the double limit is to be interpreted in the sense that $N$ never actually assumes the value $+\infty$ and $\lambda$ never actually assumes the value 1 . 
The main theorem of the paper cited in footnote 1 is the special case of this theorem for $A=0$ and much of the argument given in that paper remains the same. In particular, it was shown that the difference $D\left(x_{0}\right)$ between the functional $F(x)$ and its Fourier-Hermite partial sum is given by

$D\left(x_{0}\right)=c_{\lambda} \int_{C}^{w}\left[F(x)-F\left(x_{0}\right)\right] \exp \left\{\sum_{i=1}^{N} \frac{2 \lambda u_{i} \xi_{i}-\lambda^{2}\left(\stackrel{2}{u}_{i}+\stackrel{2}{\xi}_{i}\right)}{1-\lambda^{2}}\right\} d_{w} x$

where

$$
\begin{aligned}
c_{\lambda} & =\left(1-\lambda^{2}\right)^{-N / 2} ; \quad u_{i}=\int_{0}^{1} \alpha_{i}(t) d x(t) ; \\
\xi_{i} & =\int_{0}^{1} \alpha_{i}(t) d x_{0}(t) ; \text { and } \alpha_{i}(t)=2^{1 / 2} \cos \left(\frac{2 i-1}{2}\right) \pi t, i=1,2, \cdots
\end{aligned}
$$

We sketch here the method which was used to show domination of $D\left(x_{0}\right)$ by a sum of integrals $\sum_{j=0}^{\infty} I_{j}$.

From continuity of $F(x)$ at $x_{0} \in C$ in the Hilbert topology, it follows that if $\epsilon>0$, there exists a $\delta>0$ such that

$$
\left|F(x)-F\left(x_{0}\right)\right|<\frac{\epsilon}{3} \text { when } \int_{0}^{1}\left[x(t)-x_{0}(t)\right]^{2} d t \leqq \delta^{2} .
$$

We denote by $S_{0}$ the set of $x(t)$ satisfying this last inequality:

$$
S_{0}=\underset{x(t)}{E}\left\{\int_{0}^{1}\left[x(t)-x_{0}(t)\right]^{2} d t \leqq \delta^{2}\right\},
$$

and set

$$
\mu=\frac{\delta}{2}\left(1+\int_{0}^{1} x_{0}^{2}(t) d t\right)^{-1 / 2}
$$

then choose $N_{0}$ so large that

$$
\sum_{j=N_{0}+1}^{\infty}\left(\int_{0}^{1} \beta_{j}(t) x_{0}(t) d t\right)^{2}<\frac{\delta^{2}}{16}
$$

where

$$
\beta_{j}(t)=2^{1 / 2} \sin \left(\frac{2 j-1}{2}\right) \pi t, \quad i=1,2, \cdots
$$

Now take 


$$
\begin{aligned}
& x_{1}(t)=\sum_{j=1}^{N_{0}} \beta_{j}(t) \int_{0}^{1} \beta_{j}(s) x_{0}(s) d s ; \\
& x_{2}(t)=x_{0}(t)-x_{1}(t)
\end{aligned}
$$

and

$$
\xi_{j}^{\prime}=\int_{0}^{1} \alpha_{j}(t) d x_{2}(t)
$$

with

$$
\begin{aligned}
S_{j} & =\underset{x(t)}{E}\left\{\left|\int_{0}^{1} \alpha_{j}(t) d x(t)-\xi_{j}\right|\right. \\
& \left.>\frac{\delta}{3}\left(j-\frac{1}{2}\right)^{1 / 4}+\frac{\mu}{2}\left|\xi_{j}\right|+\left|\xi_{j}{ }^{\prime}\right|\right\}, \quad i=1,2, \cdots ;
\end{aligned}
$$

and

(4) $I_{j}=c_{\lambda} \int_{S_{j}}^{w}\left|F(x)-F\left(x_{0}\right)\right| \exp \left\{\sum_{i=1}^{N} \frac{2 \lambda u_{i} \xi_{i}-\lambda^{2}\left(\stackrel{2}{u}_{i}+\stackrel{2}{\xi_{i}}\right)}{1-\lambda^{2}}\right\} d_{w} x$;

then it was shown that

$$
\left|D\left(x_{0}\right)\right| \leqq \sum_{j=0}^{\infty} I_{j}
$$

It follows, then, from (2) and (4) that $I_{0}<\epsilon / 3$, since the boundedness of $F(x)$ did not enter into this argument.

2. General estimation of $I_{j}$. We now continue the proof of the main theorem of this paper with a consideration of $I_{j}$ for $j \neq 0$, and note that from here on the fact that $A \neq 0$ makes a difference in the details of the proof.

We shall (without loss of generality) assume that $A$ and $B$ of (1) are so chosen that

$$
\begin{aligned}
& A>1 / 8, \\
& B \geqq\left|F\left(x_{0}\right)\right| .
\end{aligned}
$$

Then by the Parseval expansion of $x(t)$,

$$
\left|F(x)-F\left(x_{0}\right)\right| \leqq 2 B \exp \left\{A \sum_{j=1}^{\infty}\left[\int_{0}^{1} \beta_{j}(t) x(t) d t\right]^{2}\right\}
$$

It follows from this and Weiner's formula 


$$
\begin{aligned}
\int_{c}^{w} f & \left\{\int_{0}^{1} \alpha_{1}(t) d x(t), \cdots, \int_{0}^{1} \alpha_{n}(t) d x(t)\right\} d_{w} x \\
& =\pi^{-n / 2} \int_{-\infty}^{\infty}(n) \int_{-\infty}^{\infty} f\left(u_{1}, \cdots, u_{n}\right) \exp \left\{-\sum_{k=1}^{n} u_{k}^{2}\right\} d u_{1} \cdots d u_{n},
\end{aligned}
$$

that the $I_{j}$ for the unbounded functional satisfy the following inequality, with $A^{\prime}=A / \pi^{2}$ :

$$
\begin{aligned}
I_{j} \leqq & 2 c_{\lambda} B \int_{s_{j}}^{w} \exp \left\{\sum_{i=1}^{N} \frac{2 \lambda u_{i} \xi_{i}-\lambda^{2}\left(u_{i}^{2}+\xi_{i}^{2}\right)}{1-\lambda^{2}}\right. \\
& \left.+A^{\prime} \sum_{i=1}^{\infty} \frac{u_{i}^{2}}{(i-1 / 2)^{2}}\right\} d_{w} x \\
= & \lim _{r \rightarrow \infty} 2 c_{\lambda} B \int_{s_{j}}^{\infty} \exp \left\{\sum_{i=1}^{N}\left[\frac{2 \lambda u_{i} \xi_{i}-\lambda^{2}\left(u_{i}^{2}+\xi_{i}^{2}\right)}{1-\lambda^{2}}+\frac{A^{\prime} u_{i}^{2}}{(i-1 / 2)^{2}}\right]\right. \\
& \left.+A^{\prime} \sum_{i=N+1}^{r} \frac{u_{i}^{2}}{(i-1 / 2)^{2}}\right\}_{w} x \\
= & 2 c_{\lambda} B \lim _{r \rightarrow \infty} \pi^{-r / 2} \int_{-\infty}^{\infty}\left(\frac{(r)}{{ }^{2}} \int_{-\infty}^{\infty} \chi_{j}\left(v_{j}\right)\right. \\
& \cdot \prod_{i=1}^{N} \exp \left\{\frac{2 \lambda u_{i} \xi_{i}-\lambda^{2}\left(v_{i}^{2}+\xi_{i}^{2}\right)}{1-\lambda^{2}}+\frac{A^{\prime} v_{i}^{2}}{(i-1 / 2)^{2}}-v_{i}^{2}\right\} \\
& \cdot \prod_{i=N+1}^{r} \exp \left\{\frac{A^{\prime} v_{i}^{2}}{(i-1 / 2)^{2}}-v_{i}^{2}\right\} d v_{1} \cdots d v_{r},
\end{aligned}
$$

where

$$
x_{j}(v)=\left\{\begin{array}{l}
1, \text { if }\left|v-\xi_{j}\right|>\frac{\delta}{3}\left(j-\frac{1}{2}\right)^{1 / 4}+\frac{\mu}{2}\left|\xi_{j}\right|+\left|\xi_{j}^{\prime}\right| ; \\
0, \text { otherwise. }
\end{array}\right.
$$

We note, for future reference, that

$$
\xi_{j}^{\prime}=\left\{\begin{array}{ll}
0, & \text { if } j \leqq N_{0} \\
\xi_{j}, & \text { if } j>N_{0}
\end{array}\right\},
$$

where $N_{0}$ is given by (3). Take $N>N_{0}$. As in the paper cited in footnote 1 we have two cases to consider, depending on $j$.

3. Estimation of $I_{j}$ for the case $j \leqq N$. Here we refine our estimate 
for $I_{j}$ for $j \leqq N$. Then

$$
\begin{aligned}
I_{j} \leqq & \frac{2 c_{\lambda} B}{\pi^{1 / 2}} \int_{-\infty}^{\infty} \chi_{j}\left(v_{j}\right) \exp \left\{\frac{2 \lambda v_{j} \xi_{j}-\lambda^{2}\left(v_{j}^{2}+\xi_{j}^{2}\right)}{1-\lambda^{3}}\right. \\
& \left.+\frac{A^{\prime} v_{j}^{2}}{(j-1 / 2)^{2}}-v_{j}^{2}\right\} d v_{j} \\
& \cdot \prod_{i=1, i \neq j}^{N}\left[\frac { 1 } { \pi ^ { 1 / 2 } } \int _ { - \infty } ^ { \infty } \operatorname { e x p } \left\{\frac{2 \lambda v_{i} \xi_{i}-\lambda^{2}\left(v_{i}^{2}+\xi_{i}^{2}\right)}{1-\lambda^{2}}\right.\right. \\
& \left.+\frac{A^{\prime} v_{i}^{2}}{(i-1 / 2)^{2}}-v_{i}^{2}\right\} d v_{i} \\
& \cdot \lim _{r \rightarrow \infty} \prod_{i=N+1}^{r} \frac{1}{\pi^{1 / 2}} \int_{-\infty}^{\infty} \exp \left\{\frac{A^{\prime} v_{i}^{2}}{(i-1 / 2)^{2}}-v_{i}^{2}\right\} d v_{i} .
\end{aligned}
$$

(6)

This last limit becomes, if

(7)

$$
N>\left(\left(A^{\prime}\right)^{1 / 2}+1\right) \text {, }
$$

$$
\lim _{r \rightarrow \infty} \prod_{i=N+1}^{r} \frac{1}{\left(1-\frac{A^{\prime}}{(i-1 / 2)^{2}}\right)^{1 / 2}}
$$

$$
=\left[\prod_{i=N+1}^{\infty}\left(1-\frac{A^{\prime}}{(i-1 / 2)^{2}}\right)\right]^{-1 / 2} .
$$

We shall need certain estimates related to the error function and we consider this next. Now

$$
\begin{aligned}
\int_{0}^{\infty} e^{-\alpha v^{2}+\beta v+\gamma} d v & =e^{\gamma+\beta^{2} / 4 \alpha} \int_{0}^{\infty} e^{-\alpha(v-\beta / 2 \alpha)^{2}} d v \\
& =e^{\gamma+\beta^{2} / 4 \alpha} \frac{1}{\alpha^{1 / 2}} \operatorname{erfc}(\Theta),
\end{aligned}
$$

where

$$
\Theta=\theta \alpha^{1 / 2}-\frac{\beta}{2 \alpha^{1 / 2}}
$$

Hence, if we take

$$
\alpha=\frac{1}{1-\lambda^{2}}-\frac{A^{\prime}}{(i-1 / 2)^{2}}, \quad \beta=\frac{2 \lambda \xi}{1-\lambda^{2}}, \quad \gamma=\frac{-\lambda^{2} \xi^{2}}{1-\lambda^{2}},
$$


we have for one of the integrals to be evaluated in (6)

$$
\begin{aligned}
& \frac{1}{\pi^{1 / 2}} \int_{-\infty}^{\infty} \exp \left\{\frac{2 \lambda v \xi-\lambda^{2}\left(v^{2}+\xi^{2}\right)}{1-\lambda^{2}}+\frac{A^{\prime} v^{2}}{(i-1 / 2)^{2}}-v^{2}\right\} d v \\
& =\exp \left\{\frac{A^{\prime} \lambda^{2} \xi^{2}}{(i-1 / 2)^{2}-A^{\prime}\left(1-\lambda^{2}\right)}\right\}\left(\frac{\left(1-\lambda^{2}\right)(i-1 / 2)^{2}}{(i-1 / 2)^{2}-A^{\prime}\left(1-\lambda^{2}\right)}\right)^{1 / 2}
\end{aligned}
$$

and for another

$$
\begin{aligned}
& \frac{1}{\pi^{1 / 2}} \int_{-\infty}^{\infty} \chi_{j}(v) \exp \left\{\frac{2 \lambda v \xi_{j}-\lambda^{2}\left(v^{2}+\xi_{j}^{2}\right)}{1-\lambda^{2}}-\frac{A^{\prime} v^{2}}{(j-1 / 2)^{2}}-v^{2}\right\} d v \\
& =\exp \left\{\frac{A^{\prime} \lambda^{2} \xi_{j}^{2}}{(i-1 / 2)^{2}-A^{\prime}\left(1-\lambda^{2}\right)}\right\}\left(\frac{\left(1-\lambda^{2}\right)(j-1 / 2)^{2}}{(j-1 / 2)^{2}-A\left(1-\lambda^{2}\right)}\right)^{1 / 2} \\
& \cdot \frac{1}{\pi^{1 / 2}}\left[\operatorname{erfc}\left(\Theta_{j}^{\prime}\right)+\operatorname{erfc}\left(-\Theta_{j}^{\prime \prime}\right)\right]
\end{aligned}
$$

where $\Theta_{j}^{\prime}$ and $\Theta_{j}^{\prime \prime}$ are related to $\theta_{j}^{\prime}$ and $\theta_{j}^{\prime \prime}$ respectively as in (9), and

$$
\begin{aligned}
& \theta_{j}^{\prime}=\xi_{j}+\frac{\delta}{3}\left(j-\frac{1}{2}\right)^{1 / 4}+\frac{1}{2} \mu\left|\xi_{j}\right|+\left|\xi_{j}^{\prime}\right|, \\
& \theta_{j}^{\prime \prime}=\xi_{j}-\frac{\delta}{3}\left(j-\frac{1}{2}\right)^{1 / 4}-\frac{1}{2} \mu\left|\xi_{j}\right|-\left|\xi_{j}^{\prime}\right| .
\end{aligned}
$$

Putting (10), (11), and (8) in (6), we obtain, when $A^{\prime}\left(1-\lambda^{2}\right)<1 / 4$ and (7) holds,

$$
\begin{aligned}
I_{j} \leqq & 2 c_{\lambda} B\left(1-\lambda^{2}\right)^{N / 2} \prod_{i=1}^{N}\left[1-\frac{A^{\prime}\left(1-\lambda^{2}\right)}{(j-1 / 2)^{2}}\right]^{-1 / 2} \\
& \cdot \exp \left\{A^{\prime} \lambda^{2} \sum_{i=1}^{\infty} \frac{\xi_{i}^{2}}{(i-1 / 2)^{2}-A^{\prime}\left(1-\lambda^{2}\right)}\right\} \\
& \cdot \frac{1}{\pi^{1 / 2}}\left\{\operatorname{erfc}\left(-\Theta_{j}^{\prime \prime}\right)+\operatorname{erfc}\left(\Theta_{j}^{\prime}\right)\right\} \\
& \cdot \prod_{i=N+1}^{\infty}\left(1-\frac{A^{\prime}}{(i-1 / 2)^{2}}\right)^{-1 / 2}
\end{aligned}
$$

Then if

$$
1>\lambda>\left(1-1 / 8 A^{\prime}\right)^{1 / 2},
$$


we have

$$
A^{\prime}\left(1-\lambda^{2}\right)<\frac{1}{8} \leqq \frac{1}{2}\left(i-\frac{1}{2}\right)^{2}, \quad i=1,2, \cdots
$$

Hence

$$
\begin{aligned}
\sum_{i=1}^{\infty} \frac{\xi_{i}^{2}}{(i-1 / 2)^{2}-A^{\prime}\left(1-\lambda^{2}\right)} & \leqq \sum_{i=1}^{\infty} \frac{\xi_{i}^{2}}{(i-1 / 2)^{2}} \cdot \frac{(i-1 / 2)^{2}}{(i-1 / 2)^{2}-A^{\prime}\left(1-\lambda^{2}\right)} \\
& \leqq 2 \sum_{i=1}^{\infty} \frac{\xi_{i}^{2}}{(i-1 / 2)^{2}} \\
& =2 \sum_{i=1}^{\infty} \frac{\left[\int_{0}^{1} \alpha_{i}(t) d x_{0}(t)\right]^{2}}{(i-1 / 2)^{2}} \\
& =2 \pi^{2} \sum_{i=1}^{\infty}\left[\int_{0}^{1} \beta_{i}(t) x_{0}(t) d t\right]^{2} \\
& =2 \pi^{2} \int_{0}^{1}\left[x_{0}(t)\right]^{2} d t .
\end{aligned}
$$

Thus

$$
\begin{aligned}
\exp \left\{A^{\prime} \lambda^{2} \sum_{i=1}^{\infty} \frac{\xi_{i}^{2}}{(i-1 / 2)^{2}-A^{\prime}\left(1-\lambda^{2}\right)}\right\} & \leqq \exp \left\{2 A^{\prime} \pi^{2} \int_{0}^{1}\left[x_{0}(t)\right]^{2} d t\right\}=B_{1}
\end{aligned}
$$

a constant depending only on $A^{\prime}$ and $x_{0}(t)$.

Assuming

$$
N>\left(A^{\prime}\right)^{1 / 2}+1
$$

we have $N+1>\left(A^{\prime}\right)^{1 / 2}+2 \geqq\left[\left(A^{\prime}\right)^{1 / 2}\right]+2$. Hence the set of integers $i \geqq N+1$ is a subset of the set of integers $i \geqq\left[\left(A^{\prime}\right)^{1 / 2}\right]+2$. Moreover, if $i \geqq\left[\left(A^{\prime}\right)^{1 / 2}\right]+2$, we have

$$
i \geqq\left(A^{\prime}\right)^{1 / 2}+1, \text { and } i-1 / 2>\left(A^{\prime}\right)^{1 / 2},
$$

so

$$
\left[1-\frac{A^{\prime}}{(i-1 / 2)^{2}}\right]^{-1 / 2}>1
$$




$$
\begin{aligned}
\prod_{i=N+1}^{\infty}\left[1-\frac{A^{\prime}}{(i-1 / 2)^{2}}\right]^{-1 / 2} & \\
& \leqq \prod_{i=\left[\left(A^{\prime}\right)^{1 / 2}+3+2\right.}^{\infty}\left[1-\frac{A^{\prime}}{(i-1 / 2)^{2}}\right]^{-1 / 2}=B_{3}
\end{aligned}
$$

for all $N>\left(A^{\prime}\right)^{1 / 2}+1$, where $B_{3}$ is a constant dependent only on $A^{\prime}$.

It follows from (14) that

$$
1-\frac{A^{\prime}\left(1-\lambda^{2}\right)}{(i-1 / 2)^{2}}>1-\frac{1}{8(i-1 / 2)^{2}},
$$

so

$$
\begin{aligned}
\prod_{i=1}^{N}\left[1-\frac{A^{\prime}\left(1-\lambda^{2}\right)}{(i-1 / 2)^{2}}\right]^{-1 / 2} & <\prod_{i=1}^{N}\left[1-\frac{1}{8(i-1 / 2)^{2}}\right]^{-1 / 2} \\
& <\prod_{i=1}^{\infty}\left[1-\frac{1}{8(i-1 / 2)^{2}}\right]^{-1 / 2} \\
& =\left[\cos \left(\frac{\pi}{2^{3 / 2}}\right)\right]^{-1 / 2}=B_{2}
\end{aligned}
$$

where $B_{2}$ is a constant independent of $N$ and $\lambda$. By (9)

$$
\begin{aligned}
\Theta_{j}^{\prime}= & \theta_{j}^{\prime} \alpha^{1 / 2}-\frac{\beta}{2 \alpha^{1 / 2}}=\theta_{j}^{\prime}\left(\frac{1}{1-\lambda^{2}}-\frac{A^{\prime}}{(j-1 / 2)^{2}}\right)^{1 / 2} \\
& -\frac{\lambda \xi_{j}}{\left(1-\lambda^{2}\right)\left(\frac{1}{1-\lambda^{2}}-\frac{A^{\prime}}{(j-1 / 2)^{2}}\right)^{1 / 2}} \\
& =\frac{1}{\left(1-\lambda^{2}\right)^{1 / 2}}\left(1-\frac{A^{\prime}\left(1-\lambda^{2}\right)}{(j-1 / 2)^{2}}\right)^{1 / 2}\left[\theta_{j}^{\prime}-\frac{\lambda \xi_{j}}{1-\frac{A^{\prime}\left(1-\lambda^{2}\right)}{(j-1 / 2)^{2}}}\right] .
\end{aligned}
$$

Note that $1-A^{\prime}\left(1-\lambda^{2}\right) /(j-1 / 2)^{2}>1 / 2$ by (14). Then from (12)

$$
\begin{array}{r}
\Theta_{j}^{\prime}=\frac{1}{\left(1-\lambda^{2}\right)^{1 / 2}}\left(1-\frac{A^{\prime}\left(1-\lambda^{2}\right)}{(j-1 / 2)^{2}}\right)^{1 / 2}\left[\xi_{j}+\frac{\delta}{3}\left(j-\frac{1}{2}\right)^{1 / 4}\right. \\
\left.+\frac{1}{2} \mu\left|\xi_{j}\right|+\left|\xi_{j}^{\prime}\right|-\frac{\lambda \xi_{j}}{1-\frac{A^{\prime}\left(1-\lambda^{2}\right)}{(j-1 / 2)^{2}}}\right] .
\end{array}
$$

Now let $A^{\prime}\left(1-\lambda^{2}\right) /(j-1 / 2)^{2}=a$. By (14), $a<1 / 2$, so that 


$$
\begin{aligned}
\left|\frac{\lambda-1+a}{1-a}\right| & <2|\lambda-1+a|=2\left|\lambda-1+\frac{A^{\prime}\left(1-\lambda^{2}\right)}{(j-1 / 2)^{2}}\right| \\
& =2(1-\lambda)\left|\frac{A^{\prime}(1+\lambda)}{(j-1 / 2)^{2}}-1\right|<2(1-\lambda)\left(8 A^{\prime}+1\right) .
\end{aligned}
$$

Now if $\lambda$ is so chosen that

$$
1>\lambda>1-\mu / 4\left(8 A^{\prime}+1\right),
$$

it follows that

$$
\frac{1}{2} \mu>\left|\frac{\lambda-1+a}{1-a}\right| .
$$

Hence

$$
\frac{1}{2} \mu\left|\xi_{j}\right| \geqq \frac{-\xi_{j}(1-\lambda)+a \xi_{j}}{1-a}
$$

and

$$
\xi_{j}+\frac{1}{2} \mu\left|\xi_{j}\right|+\left|\xi_{j}^{\prime}\right|-\frac{\lambda \xi_{j}}{1-a} \geqq 0 .
$$

Thus

$$
\Theta_{j}^{\prime}>\frac{1}{\left(1-\lambda^{2}\right)^{1 / 2}} \cdot \frac{\delta}{3 \cdot 2^{1 / 2}}\left(j-\frac{1}{2}\right)^{1 / 4} \text {. }
$$

Again, by (9),

$$
\begin{aligned}
\Theta_{j}^{\prime \prime}= & \theta_{j}^{\prime \prime} \alpha^{1 / 2}-\frac{\beta}{2 \alpha^{1 / 2}} \\
= & \frac{(1-a)^{1 / 2}}{\left(1-\lambda^{2}\right)^{1 / 2}}\left[\xi_{j}-\frac{\delta}{3}\left(j-\frac{1}{2}\right)^{1 / 4}\right. \\
& \left.\quad-\frac{1}{2} \mu\left|\xi_{j}\right|-\left|\xi_{j}^{\prime}\right|-\frac{\lambda \xi_{j}}{1-a}\right] .
\end{aligned}
$$

Multiplying (19) by $\left|\xi_{j}\right|$ gives

$$
\begin{gathered}
\frac{1}{2} \mu\left|\xi_{j}\right|>\left|\frac{(\lambda-1) \xi_{j}+a \xi_{j}}{1-a}\right| \geqq \frac{(1-\lambda) \xi_{j}-a \xi_{j}}{1-a}=\xi_{j}-\frac{\lambda \xi_{j}}{1-a} . \\
\xi_{j}-\frac{1}{2} \mu\left|\xi_{j}\right|-\left|\xi_{j}^{\prime}\right|-\frac{\lambda \xi_{j}}{1-a} \leqq 0,
\end{gathered}
$$


and this with (20) gives

$$
\Theta_{j}^{\prime \prime} \leqq \frac{-1}{\left(1-\lambda^{2}\right)^{1 / 2}} \frac{\delta}{3 \cdot 2^{1 / 2}}\left(j-\frac{1}{2}\right)^{1 / 4} .
$$

Putting in (13) the estimates for each of the factors (17), (15), and (16), we have

$$
\begin{aligned}
I_{j} & \leqq 2 B B_{1} B_{2} B_{3} \frac{2}{\pi^{1 / 2}} \operatorname{erfc}\left\{\frac{1}{\left(1-\lambda^{2}\right)^{1 / 2}} \frac{\delta}{3 \cdot 2^{1 / 2}}\left(j-\frac{1}{2}\right)^{1 / 4}\right\} \\
& =B_{4} \int_{\delta(j-1 / 2)^{1 / 4} / 3 \cdot 2^{1 / 2}\left(1-\lambda^{2} 1 / 2\right.}^{+\infty} e^{-x} d x,
\end{aligned}
$$

our final estimate for the first case.

4. Estimation of $I_{j}$ for the case $j>N$. Since $N>N_{0}$, we also have $j>N_{0}$. Now by (5)

$$
\begin{aligned}
I_{j} \leqq & 2 c_{\lambda} B \prod_{i=1}^{N} \frac{1}{\pi^{1 / 2}} \int_{-\infty}^{\infty} \exp \cdot\left\{\frac{2 \lambda v_{i} \xi_{i}-\lambda^{2}\left(v_{i}^{2}+\xi_{i}^{2}\right)}{1-\lambda^{2}}\right. \\
& \left.+\frac{A^{\prime} v_{i}^{2}}{(i-1 / 2)^{2}}-v_{i}^{2}\right\} d v_{i} \\
& \frac{1}{\pi^{1 / 2}} \int_{-\infty}^{\infty} \chi_{j}\left(v_{j}\right) \exp \cdot\left\{\frac{A^{\prime} v_{j}^{2}}{(j-1 / 2)^{2}}-v_{j}^{2}\right\} d v_{j} \\
& \lim _{r \rightarrow \infty} \prod_{i=N+1, i \neq j}^{r}\left[\frac{1}{\pi^{1 / 2}} \int_{-\infty}^{\infty} \exp \cdot\left\{\frac{A^{\prime} v_{i}^{2}}{(i-1 / 2)^{2}}-v_{i}^{2}\right\} d v_{i}\right] .
\end{aligned}
$$

The limit above equals, if $N>\left(A^{\prime}\right)^{1 / 2}+1$, as in (15),

$$
\begin{aligned}
\lim _{r \rightarrow \infty} \prod_{i=N+1, i \neq j}^{r}\left[1-\frac{A^{\prime}}{(i-1 / 2)^{2}}\right]^{-1 / 2} & \\
= & {\left[\prod_{i=N+1, i \neq j}^{\infty}\left(1-\frac{A^{\prime}}{(i-1 / 2)^{2}}\right)\right]^{-1 / 2} . }
\end{aligned}
$$

Now set

$$
v_{j}\left(1-\frac{A^{\prime}}{(j-1 / 2)^{2}}\right)^{1 / 2}=v_{j} R_{j}=s
$$

so that

$$
R_{j}^{2}=1-A^{\prime} /(j-1 / 2)^{2}
$$


Then (23) becomes

(25) $\frac{1}{R_{j} \pi^{1 / 2}} \int_{-\infty}^{\infty} \chi_{j}\left(\frac{s}{R_{j}}\right) e^{-\theta^{2}} d s=\frac{1}{R_{j} \pi^{1 / 2}}\left(\int_{\theta \cdot R}^{\infty}+\int_{-\theta_{j}{ }^{\prime \prime} R_{j}}^{\infty}\right) e^{-\theta^{2} d s}$.

But

$$
\begin{aligned}
\theta_{j}^{\prime} & =\xi_{j}+\frac{\delta}{3}\left(j-\frac{1}{2}\right)^{1 / 4}+\frac{1}{2} \mu\left|\xi_{j}\right|+\left|\xi_{j}^{\prime}\right| \\
& =\xi_{j}+\frac{\delta}{3}\left(j-\frac{1}{2}\right)^{1 / 4}+\frac{1}{2} \mu\left|\xi_{j}\right|+\left|\xi_{j}\right| \\
& \geqq \frac{\delta}{3}\left(j-\frac{1}{2}\right)^{1 / 4},
\end{aligned}
$$

and similarly,

$$
\theta_{j}^{\prime \prime} \leqq-(\delta / 3)(j-1 / 2)^{1 / 4} .
$$

Then for $j>N>\left(2 A^{\prime}\right)^{1 / 2}+1, A^{\prime} /(j-1 / 2)^{2}<1 / 2$, and $R_{j}>1 / 2^{1 / 2}$. So the first member of (25) is

$$
\leqq \frac{2}{R_{j} \pi^{1 / 2}} \int_{\left(\delta / 3 \cdot 2^{1 / 2}\right)(j-1 / 2)^{1 / 4}}^{\infty} e^{-s^{2}} d s .
$$

The product of (23) and (24), then, is

$$
\begin{aligned}
& \leqq \frac{2}{\pi^{1 / 2}}\left(\prod_{i=N+1}^{\infty} R_{i}\right)^{-1} \operatorname{erfc}\left\{\frac{\delta}{3 \cdot 2^{1 / 2}}\left(j-\frac{1}{2}\right)^{1 / 4}\right\} \\
\text { (26) } & \leqq \frac{2}{\pi^{1 / 2}} B_{3} \operatorname{erfc}\left\{\frac{\delta}{3 \cdot 2^{1 / 2}}\left(j-\frac{1}{2}\right)^{1 / 4}\right\},
\end{aligned}
$$

since (16) still holds. Now each of the $N$ integrals in (22) is of the type already evaluated in (10), so by this and (26) we have

$$
\begin{aligned}
I_{j} \leqq & 2 c_{\lambda} B \exp \cdot\left\{A^{\prime} \lambda^{2} \sum_{i=1}^{N} \frac{\xi_{i}^{2}}{(i-1 / 2)^{2}-A^{\prime}\left(1-\lambda^{2}\right)}\right\} \\
& \cdot \prod_{i=1}^{N}\left(\frac{\left(1-\lambda^{2}\right)(i-1 / 2)^{2}}{(i-1 / 2)^{2}-A^{\prime}\left(1-\lambda^{2}\right)}\right)^{1 / 2} \\
& \cdot \frac{2}{\pi^{1 / 2}} B_{3} \operatorname{erfc}\left\{\frac{\delta}{3 \cdot 2^{1 / 2}}\left(j-\frac{1}{2}\right)^{1 / 4}\right\} \\
\leqq & \frac{4}{\pi} B B_{3} B_{1} B_{2} \operatorname{erfc}\left\{\frac{\delta}{3 \cdot 2^{1 / 2}}\left(j-\frac{1}{2}\right)^{1 / 4}\right\},
\end{aligned}
$$


by (15) and (17). Thus

$$
\begin{aligned}
I_{j} & \leqq B_{4} \operatorname{erfc}\left\{\frac{\delta}{3 \cdot 2^{1 / 2}}\left(j-\frac{1}{2}\right)^{1 / 4}\right\} \\
& =B_{4} \int_{\left(8 / 8 \cdot 2^{1 / 2}\right)(j-1 / 2)^{1 / 4}}^{\infty} e^{-v^{2} d v}
\end{aligned}
$$

This is our final estimate for the second case.

5. Final estimate for $D\left(x_{0}\right)$. We combine the estimates (21) and (27) and assume for this that $N>\max \left\{N_{0},\left(2 A^{\prime}\right)^{1 / 2}+1\right\}$, and, further, that

$$
1>\lambda>\max \left\{\left(1-\frac{1}{8 A^{\prime}}\right)^{1 / 2}, 1-\frac{\mu}{4\left(8 A^{\prime}+1\right)}\right\} .
$$

Thus (14) and (18) hold and hence the estimates (21) and (27). Thus

$$
\begin{aligned}
& \sum_{j=1}^{\infty} I_{j} \leqq B_{4}\left[\sum_{j=1}^{N} \operatorname{erfc}\left\{\frac{\delta(j-1 / 2)^{1 / 4}}{3 \cdot 2^{1 / 2}\left(1-\lambda^{2}\right)^{1 / 2}}\right\}\right. \\
&+\sum_{j=N+1}^{\infty} \operatorname{erfc}\left\{\frac{\delta(j-1 / 2)^{1 / 4}}{3 \cdot 2^{1 / 2}}\right\} .
\end{aligned}
$$

Since

$$
\begin{gathered}
\operatorname{erfc}(M)=\int_{M}^{\infty} e^{-v^{2}} d v<\frac{e^{-M^{2}}}{2 M} \quad(M>0), \\
\sum_{j=1}^{\infty} I_{j}<\frac{B_{4}}{2} \sum_{j=1}^{\infty} \frac{3 \cdot 2^{1 / 2}\left(1-\lambda^{2}\right)^{1 / 2}}{\delta(j-1 / 2)^{1 / 4}} \exp \left\{\frac{-\delta^{2}(j-1 / 2)^{1 / 2}}{18\left(1-\lambda^{2}\right)}\right\} \\
+\frac{B_{4}}{2} \sum_{j=N+1}^{\infty} \frac{3 \cdot 2^{1 / 2}}{\delta(j-1 / 2)^{1 / 4}} \exp \left\{\frac{-\delta^{2}(j-1 / 2)^{1 / 2}}{18}\right\} .
\end{gathered}
$$

Since the sums on the right converge, we may take $N_{\epsilon}$ large enough to make the last term less than $\epsilon / 3$ for $N>N_{\epsilon}$.

Moreover the first series on the right converges and decreases term by term as $\lambda \rightarrow 1-0$, and has the limit 0 . We may therefore choose $\lambda_{\epsilon}$ in the interval

$$
1>\lambda_{\epsilon}>\max \left\{\left(1-\frac{1}{8 A^{\prime}}\right)^{1 / 2}, 1-\frac{\mu}{4\left(8 A^{\prime}+1\right)}\right\},
$$

so that the first term on the right is less than $\epsilon / 3$ when $\lambda_{\epsilon}<\lambda<1$. Thus when 


$$
\begin{gathered}
N>N_{\epsilon} \text { and } \lambda_{\epsilon}<\lambda<1, \\
\left|D\left(x_{0}\right)\right| \leqq \sum_{j=0}^{\infty} I_{j}<\epsilon,
\end{gathered}
$$

by the above inequalities and (4.1) of the paper cited in footnote 1 . The same corollary as in that paper holds now for the functional of (1)-and for the same reasons as given in the proof for the bounded functional.

The University of Minnesota

\section{ESSENTIALLY ADMISSIBLE SEQUENCES}

\section{R. CREIGHTON BUCK}

Let $X$ be the set of all complex sequences $\alpha=\left\{a_{n}\right\}$ such that $\|\alpha\|=\sup _{n}\left|a_{n}\right|^{1 /(n+1)}<\infty$. Under the usual operations, $X$ is a complex vector space, and $\|\alpha+\beta\| \leqq\|\alpha\|+\|\beta\|$. However, $\|c \alpha\|$ is seldom $|c|\|\alpha\|$ and even though $c_{n} \rightarrow 0$, it is not in general true that $\left\|c_{n} \alpha\right\| \rightarrow 0$; for example, if $\alpha=\langle 1,1,1, \cdots\rangle$, then $\|c \alpha\|$ is $c$ if $c \geqq 1$ and is 1 if $0<c<1$. Defining the distance between $\alpha$ and $\beta$ as $\|\alpha-\beta\|, X$ becomes a complete metric abelian group, but not a topological linear space. If with each $\alpha$ in $X$ is associated the analytic function defined at the origin by $f(z)=\sum a_{n} z^{n}$, then this topology is that in which a sequence $\left\{f_{n}\right\}$ converges to the zero function only if on every bounded domain $D$, and for sufficiently large $n$, the functions $f_{n}$ are all analytic on $D$ and converge uniformly to zero; $f_{n}$ converges to $g$ if $f_{n}-g$ converges to zero. This topology is closely related to that introduced by Ganapathy Iyer into the vector space of all entire functions [4].

Given any $\alpha \in X$, there may be found an entire function $f(z)$ of order 1 , finite type, and such that $f(i y)=O(\exp c|y|)$ for some $c \leqq \pi$, which interpolates to $\alpha$ in the sense that $f(n)=a_{n}$ for $n=0,1,2, \ldots$ [1]. This is not the case if the condition $c<\pi$ is imposed. We have called a sequence $\alpha$ admissible in case such a more restricted function exists [2]. By a theorem of Carlson, such a function when it exists is unique [3]. A sequence $\alpha$ may fail to be admissible in an inessential way; for example, $\alpha=\langle 0,0,0, \ldots\rangle$ is admissible, but $\beta$ $=\langle 1,0,0, \cdots\rangle$ is not. To allow for this, we shall now say that $\alpha$

Presented to the Society, September 4, 1952; received by the editors October 16, 1952. 